Review

\title{
Roles of DLK1 in Liver Development and Oncogenesis
}

\author{
${ }_{1,2,3}$ Ahmad Azmi Nasution, ${ }^{1,2,4}$ Msy Rulan Adnindya, \\ ${ }^{1,2,5}$ Indriyani and ${ }^{1,2,6}$ Isabella Kurnia Liem \\ ${ }^{I}$ Department of Anatomy, Faculty of Medicine, Universitas Indonesia, Jakarta, Indonesia \\ ${ }^{2}$ Master Program in Biomedical Sciences, Faculty of Medicine, Universitas Indonesia, Jakarta, Indonesia \\ ${ }^{3}$ Department of Anatomy, Faculty of Medicine and Health Science, Universitas Bengkulu, Bengkulu, Indonesia \\ ${ }^{4}$ Department of Anatomy, Faculty of Medicine, Sriwijaya University, Palembang, Indonesia \\ ${ }^{5}$ Department of Anatomy, Faculty of Medicine, Universitas Muhammadiyah Palembang, Palembang, Indonesia \\ ${ }^{6}$ Department of Anatomy, Faculty of Medicine, Universitas Indonesia, Jakarta, Indonesia
}

\author{
Article history \\ Received: 05-03-2017 \\ Revised: 02-06-2017 \\ Accepted: 29-09-2017 \\ Corresponding Author: \\ Isabella Kurnia Liem \\ Department of Anatomy, \\ Faculty of Medicine, \\ Universitas Indonesia, Jakarta, \\ Indonesia \\ Email: bellajo04@gmail.com
}

\begin{abstract}
Hepato Cellular Carcinoma (HCC) has an increased mortality rate in the last decade. Protein expression in HCC has similarities to the liver cells during the development process. One of the proteins expressions is Delta-Like 1 homolog (DLK1) that was found during liver development and oncogenesis. This permits the opportunity to study pathophysiology and signaling pathway of DLK1 and to find an early detection and therapeutic target for HCC. This review will explain the signalling mechanism and the roles of DLK1 during liver development, oncogenesis of HCC, as a tumor marker and targeting therapy.
\end{abstract}

Keywords: Hepatocellular Carcinoma, DLK1, Liver Development, HCC Oncogenesis, Tumor Marker

\section{Introduction}

Hepatocellular Carcinoma (HCC) is one of malignancies which has high mortality rate. American Cancer Society has reported an increase in the mortality rate since 2003 until 2012 as much as $2.7 \%$ due to this illness (ACS, 2016). Hepatocellular carcinoma and liver cirrhosis are the main cause of chronic hepatitis B and hepatitis $\mathrm{C}$ virus that made patients death. Approximately 1.45 million people are infected by these viruses (WHO, 2016). Because of the HCC incidence and high in mortality, it becomes a concern of researcher to make diagnostic and effective therapeutic strategies. It requires a comprehensive knowledge of liver development to understand the preventive measures, causes determination and pathogenesis of liver diseases (Sokol, 2002). One of the proteins target in liver oncogenesis is Delta-Like 1 homolog (DLK1).

Delta-Like 1 homolog (DLK1) is a candidate biomarker of liver stem/progenitor cells (Wang and Sul, 2006; Bujak et al., 2015; Kopan and Ilagan, 2009) and plays a significant role in HCC oncogenesis (Baladron et al., 2005; Laborda et al., 1993; Nueda et al., 2007). DLK $1^{+}$HCC cells have the same characteristics as cancer stem/progenitor cells. However, molecular mechanism of HCC is still not yet well-established
(Falix et al., 2012; Xu et al., 2012). This review explains the roles of DLK1 in the process of liver development, HCC oncogenesis, as a tumor marker and targeting therapy.

\section{Delta-Like 1 Homolog}

Delta-like 1 homolog is a transmembrane protein that has three major regions, i.e., an extracellular region composed of six EGF-like (epidermal growth factor like), a juxtamembrane region with TACE cleavage site (ADAM17) and an intracellular region (Bujak et al., 2015; Wang and Sul, 2006). The repeated structure of amino acid sequences of EGF are very similar to the structure of Delta-Like canonical Ligand (DLL) (Baladron et al., 2005; Nueda et al., 2007; Bujak et al., 2015); however, DLK1 does not have N-terminal Delta-Serrate-LAG-2 (DSL)-domain (Yin et al., 2006; Kopan and Ilagan, 2009). Consequently, Delta and OSM-11-like proteins (DOS) co-ligand functioned to inhibit NOTCH signalling. DOS is a specialized tandem of EGF-repeats (Baladron et al., 2005; Kopan and Ilagan, 2009; Nueda et al., 2007; Fig. 1).

DLK1 shows inhibitory activity in NOTCH signalling pathway (Kopan and Ilagan, 2009). It is expressed widely during embryonic development and controls the determination process of cell fate, proliferation and differentiation (Baladron et al., 2005; Falix et al., 2012; Nueda et al., 2007; Fig. 1). 


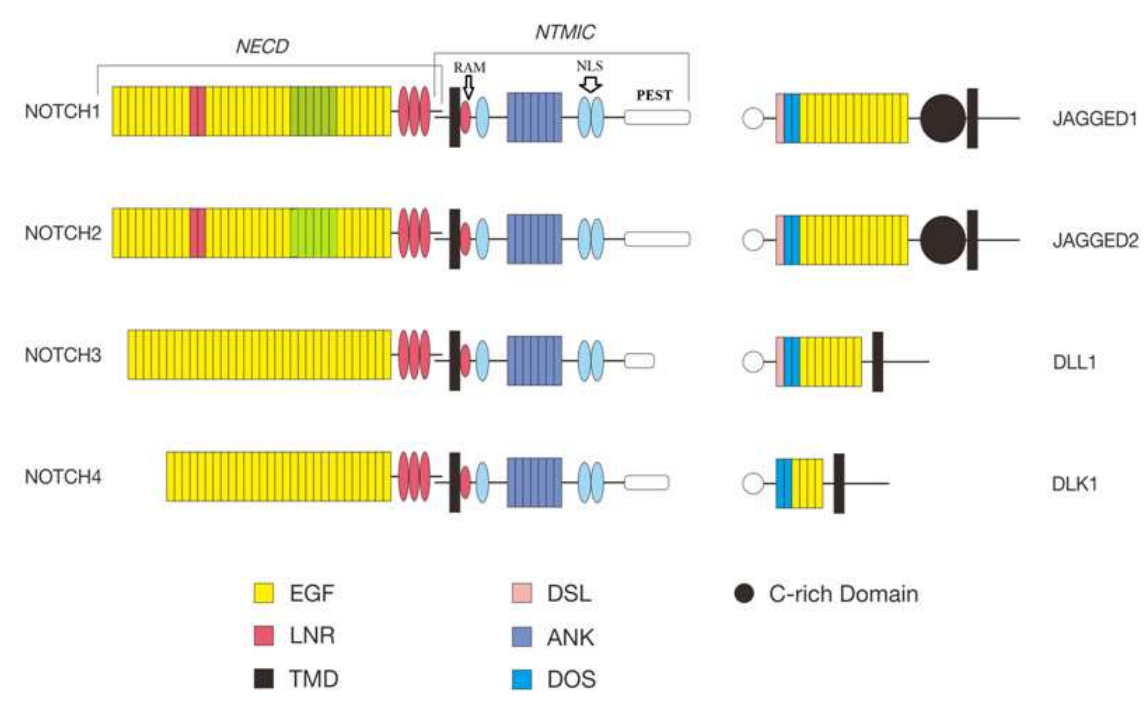

Fig. 1. Schematic diagram of NOTCH1, NOTCH2, NOTCH3 and NOTCH4 receptors and their four ligands (JADDED1, JADDED2, DLL1 and DLK1). NOTCH receptors consist of multiple extracellular EGF-like repeats, which are different in the repeating number in each receptor. NOTCH receptors have two domains, i.e., extracellular (NECD) and transmembrane-intracellular (NTMIC). Ligand binding will release intracellular (NICD) fragment and leads to NOTCH signalling activity. Two NOTCH receptor ligands family are Jagged and DLL ligands. Those canonical ligands contain DSL, DOS and EGF motifs. DLK1 is a non-canonical ligand that lacks of its DSL domain; consequently, it will acts as DOS ligand

\section{DLK1-NOTCH Relationship}

In determining the fate of hepatoblast cells and HCC pathogenesis, NOTCH signaling has a significant role (Lu et al., 2016). NOTCH signalling is also required for cell commitment, cell spesification and maintenance process of progenitor cells during prenatal development (Chen et al., 2011; Dill et al., 2013; Lu et al., 2016; Loomes et al., 2002). NOTCH has four receptor types, i.e., NOTCH1-4 and two families ligands (Jagged and Delta-like) (Falix et al., 2012).

NOTCH receptors consist of two domains, i.e. NOTCH Extracellular Domain (NECD) and NOTCH Transmembrane-Intracellular domain (NTMIC) (Kopan and Ilagan, 2009; Falix et al., 2012). NOTCH extracellular domain is composed of varian EGF like repeats (29-36 EGF-like repeats), a region that mediates interaction between the receptor and its ligand, especially at EGF-like repeats 11-12 and 24-29 (Kopan and Ilagan, 2009). Epidermal growth factor repeats are followed by negative regulatory region or NRR that contains three cysteine-rich Lin12-Notch Repeats (LNR) and a heterodimerization domain or HD. In the absence of ligand, NRR has a special function to prevent receptor activation (Kopan and Ilagan, 2009; Falix et al., 2012; Teodorczyk and Schmidt, 2014).

NOTCH Transmembrane and Intracellular domain (NTMIC) are composed of transmembrane domain or TMD, a RBP-JK association module-domain for binding with DNA binding protein CSL (CBF1/RBP-J//Su(H)/Lag1) (Kopan and Ilagan, 2009), nuclear localization sequences or NLSs, a seven ankyrin reputation-domain that functions to take the coactivator Mastermind/Lag-3 and a
Transactivation Domain (TAD) that habors a conserved proline/glutamicacid/serine/threonine-rich (PEST) motifs (Teodorczyk and Schmidt, 2014; Kopan and Ilagan, 2009; Geisler et al., 2008). Glutamine-rich repeat (OPA) in Drosophila has a function as transactivation domain (Kopan and Ilagan, 2009).

Two families of ligand have three structural motifs, i.e. an N-terminal DSL motif, a specialized tandem EGF repeats, i.e. DOS-domain and variable EGF-like repeats (Falix et al., 2012; D'Souza et al., 2010; Teodorczyk and Schmidt, 2014). The difference between Jagged/Serrate ligand and Delta-like ligand are based on the existence of a cysteine-rich domain. Jagged/Serrate ligand has cysteine-rich domain and Delta-like ligand do not have it (Fig. 1). N-terminal DSL and DOS region will bind with NOTCH receptor (Kopan and Ilagan, 2009; Falix et al., 2012).

NOTCH signalling pathway begins with the binding of ligand-receptor that induces the receptor. With the aid of proteolytic enzyme, NOTCH receptor releases NICD. In further, NICD enters nucleus to bind with CBF1/RBP$\mathrm{J} \kappa$. It will activate target genes, such as Hes (hairy and enhancer of split homologs; Fig. 2) that has functions in the regulation of proliferation, differentiation and apoptosis of epithelial cells and carcinogenesis (Falix et al., 2012; Hansson et al., 2004; Lu et al., 2016). NOTCH signaling also controls Sox9, HNF1, TGF- $\beta$ and Homeobox B expressions in the liver, which play a role in determining liver cells commitment (Dill et al., 2013; Zong et al., 2009; Zong and Stanger, 2011; Figure 2). The absence of RBP-Jא indicates blocking of NOTCH signalling pathway (Morell et al., 2013). 


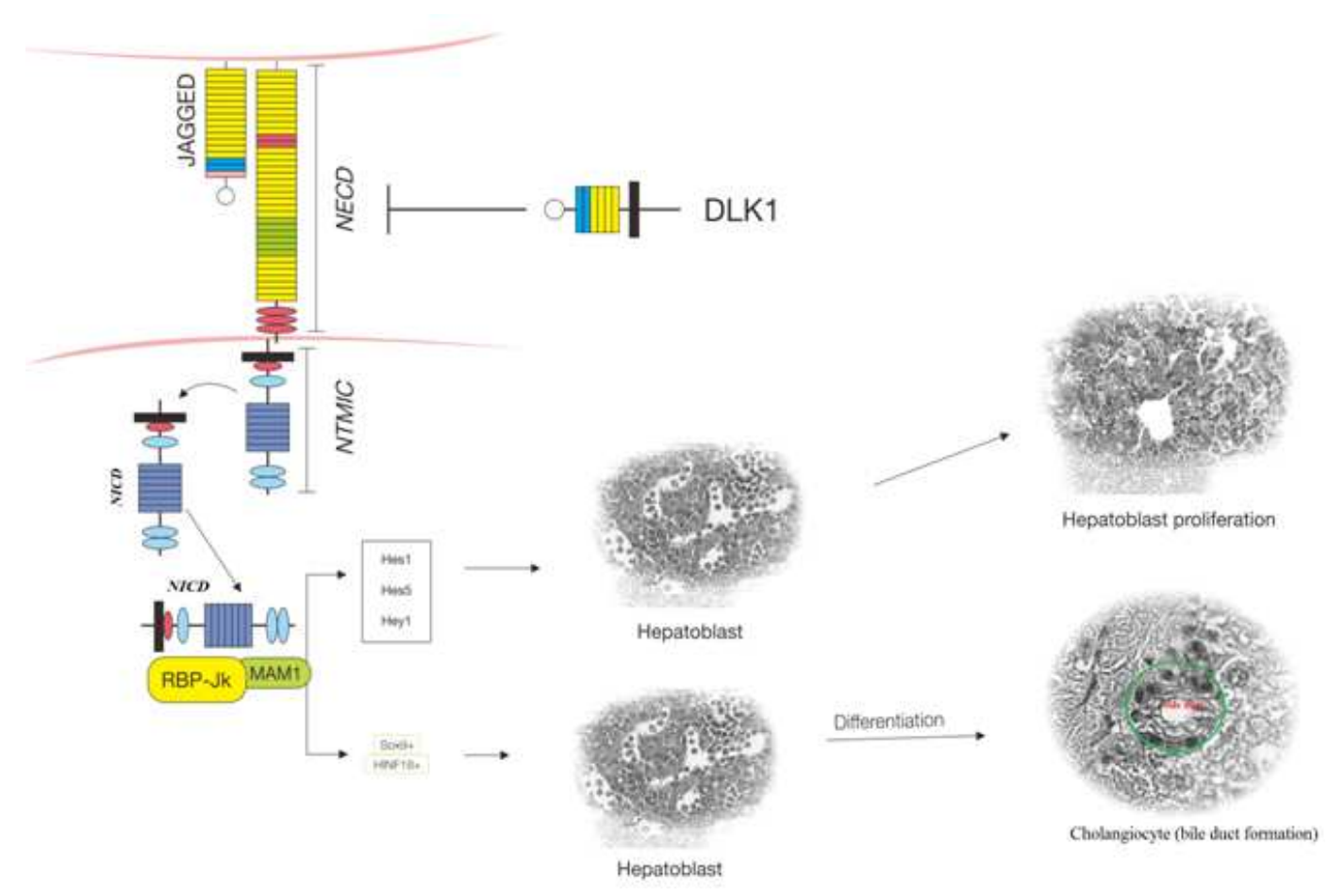

Fig. 2. DLK1 role in the proliferation of liver cells during the development is as the inhibit differentiation of hepatoblast into cholangiocyte. NOTCH receptor-canonical ligand (Jagged) binding will release NICD. After that, NICD enters the nucleus and binds with RBP- $\mathrm{J} \kappa$ to become a complex protein. This complex protein recruits MAM1 and other co-activators to activate target genes (Hes and Hey) transcription that facilitate hepatoblast differentiation into cholangiocyte. DLK1 shows inhibitory activity at the extracellular domain of NOTCH signalling pathway

In mammals, four NOTCH receptors have their own roles. NOTCH1 has a role in the normal prenatal development; nevertheless, it also involved in the regulation of oncogenesis process in adult (Falix et al., 2012). NOTCH2 is responsible for the cholangiocytes differentiation, aggressive behavior and immature morphology of HCC (Falix et al., 2012; Hayashi et al., 2015). NOTCH3 and NOTCH4 have functions in vascular development (Falix et al., 2012). DLK1 role as non-canocical ligand is as inhibitors of $\mathrm{NOTCH}$ signaling (Figure 2). It can be seen from the fact that increased DLK1 expression is associated with the decreased of NOTCH and Heslexpressions (Chen et al., 2011; Tanimizu and Mitaka, 2014).

\section{DLK1-NOTCH in Liver Development}

DLK1 has dynamic expression during liver development. DLK1 expression begins since ED10.5 and increases significantly at ED14.5-ED16.5. DLK1 expression is downregulated concomitantly with the formation of cholangiocytes and the remodelling of ductal plate into intrahepatic ducts. Eventually, DLK1 expression is no longer found after neonate period (Falix et al., 2013; Tanimizu et al., 2003; Tanaka et al., 2009).

DLK1 is a marker for hepatoblast that has high proliferation and bipotentiality. DLK1 is also considered as a marker for immature hepatocytes because DLK $1^{+}$cells express HNF1 $\beta$, HNF3 $\beta$, HNF4 and HNF6, but do not express CK19 that is expressed by cholangiocytes (Tanimizu et al., 2003; 2004). While DLK1 is down regulated, NOTCH expression is increased and at the same time hepatoblasts differentiates into cholangiocytes (Baladron et al., 2005; Gordillo et al., 2015; Loomes et al., 2002; Yin et al., 2006).

NOTCH signalling is responsible for activation of target genes, i.e., Hes, Sox9, HNF1 $\beta$, that are important for cholangiocytes differentiation (Gordillo et al., 2015; Tanimizu and Mitaka, 2014; Shin et al., 2015; Jörs et al., 2015; Morell et al., 2013; Fig. 2). In addition, DLK1 also has a role for progenitor/stem cells' maintenance and differentiation during development (Begum et al., 2014). DLK1-Wnt10b- $\beta$ Catenin pathway contributes in hepatocytes proliferation during liver regeneration. DLK $1^{+}$hepatocyte cells are induced by hepatic stellate cells through paracrine effects and selfinductive (Zhu et al., 2012).

\section{DLK1-NOTCH Role in Hepatocellular Carcinoma Oncogenesis}

Some spectrum of tumors, such as breast cancer, small-cell lung carcinoma, leukemia, neuroblastoma, 
gliomas, pancreatic cancer, colon cancer, have high DLK1 expression (Begum et al., 2014; Bujak et al., 2015). In the pathogenesis of liver diseases, NOTCH signaling has a significant role. Increased expression of NOTCH3 and NOTCH4 are found in cancer tissues. Observation of HCC HepG2 cell lines showed a relatively high $\mathrm{NOTCH} 3$ expression and a little expression of NOTCH4 (Geisler et al., 2008). In the pathogenesis of $\mathrm{HCC}$, there is a mechanism involving DLK1 and NOTCH signaling pathway. Regulation of HCC is through multiple signaling pathways including NOTCH, RAF/MEK/ERK, Wnt/ק-catenin, AKT/mTOR, EGFR, HGF/cMET and (Coral et al., 2012; Woo et al., 2009; Zhao et al., 2016; Fig. 3).

DLK1 expression can be induced by hypoxia; therefore, it is possible that oncogenesis process involving DLK1 were affected by hypoxic conditions in the microenvironment (Kim et al., 2009). DLK1 is also secreated in hepatic stellate cells selectively and will induce the activation of hepatic stellate cells in vivo and in vitro (Zhu et al., 2012). DLK1 will upregulate WNT pathway. Knockdown of DLK1 will lead to the reduction of Wnt10b, Wnt3a, necdin and Shh expression (Zhu et al., 2012). In vitro, DLK1 inhibit Mesenchymal Stem Cells (MSC) differentiation (Chen et al., 2011). In vivo, overexpression of DLK1 will increase the stemness of the tumor cell and tumor growth (Begum et al., 2014; Kim et al., 2009). $\mathrm{DLK}^{+} \mathrm{HCC}$ showed to form a colony, spheroid colony and higher chemoresistency compared to DLK1 knockdown on HCC cells (Xu et al., 2012).

Another oncogenesis process of HCC is by activation of NOTCH signaling pathway that will lead an epithelial tranformation into mesenchymal (Fig. 3). This change will also result in the disappearance of main characteristic of epithel cells and play a role in embryonic development, liver fibrosis and cancer (Zhao et al., 2016).

NOTCH2 signalling can lead and accelareated HCC formation that has the same characterictics and expression pattern as hepatoblast (Hayashi et al., 2015; Dill et al., 2013), but with great migration and invasion capability (Gordillo et al., 2015).

NOTCH2 signalling is one of key regulators in HCC. NOTCH2 signalling will increase Hes1 and Sox9 mRNA expression related to HCC formation (Tanimizu et al., 2003; Dill et al., 2013). Increased proliferation on DEN-N2ICD mice correlated to the increased of NOTCH2 espression. The increased of Hes1 mRNA will eventually form a colony in HCC formation. In addition, NOTCH2 signalling will accelerate the HCC growth (Dill et al., 2013).

In the last 5 years, researches have demonstrated the role of hepatic stellate cells and some morphogensin oncogenesis process of HCC (Zhu et al., 2012; Fung et al., 2016). Activated hepatic stellate cells will express some morphogen such as Wnt, Necdin, DLK1, Shh and NOTCH. Wnt pathway, Necdin and DLK1 have a function in $\mathrm{HCC}$ through the regulatory role of $\mathrm{PPAR}_{\gamma}$. $\mathrm{PPAR}_{\gamma}$ plays a role in inhibiting tumor growth and invasion of cells to the surrounding tissues. Another function of PPAR $\gamma$ is to inhibit EMT process which plays a role in HCC (Fung et al., 2016; Hsu and Chi 2014; Kimura et al., 2012; Shen et al., 2012; Zhu et al., 2012). Studies that have been conducted gave indirect evidence to the relation of Wnt, Necdin, DLK1 and PPAR $\gamma$ in HCC. Necdin has a role in activation of Wnt pathway by binding with GN boxes on the proximal promoter of Wnt10b resulting in the repression of PPAR $\gamma$. This proves that there is an opposite role of Necdin and Wnt with PPARy (Ross et al., 2000; Zhu et al., 2012). In studies of DLK1 knockdown, induction of PPAR $\gamma$ was occured. It was almost identical with Necdin and Wnt mechanism in regulation of PPAR $\gamma$ (Zhu et al., 2012; Fung et al., 2016). Therefore, the increase of Wnt, Necdin and DLK1 expression will lead to PPAR $\gamma$ suppression resulting in increased expression of HGF, which has a role in EMT that will eventually develop into HCC (Fung et al., 2016; Maulik et al., 2002; Ding et al., 2010; Mizuno et al., 2005; Ozaki et al., 2003). Additionally, proliferation and invation of $\mathrm{HCC}$ will be increased due to $\mathrm{HGF}$ stimulation of Matrix metallopeptidase 9 (MMP9) and Matrix metallopeptidase 3 (MMP3) (Wang et al., 2007; Ozaki et al., 2003; Mizuno et al., 2005; Mohammed et al., 2005; Lee et al., 2010).

\section{DLK1 as Hepatocellular Carcinoma Marker}

Delta-like 1 homolog is expressed in malignancy and promotes cancer cell stemness and tumorigenicity so it can be used as therapeutic target and tumor marker for cancer stem/progenitor cells. Li et al. (2015) revealed that tumor size of HCC was positively correlated to DLK1 in serum. DLK1 can be a complement to Alpha Feto Protein (AFP) in the diagnosis of HCC (Li et al., 2015; Chauhan and Lahiri 2016; Shen et al., 2016).

It is possible to use DLK1 as a prognostic factor since there is an evidence that it correlates to survival rate in $\mathrm{HCC}$. $\mathrm{HCC}$ patients with $\mathrm{DLK}^{+}$cells have shorther survival rate compared to HCC patients without $\mathrm{DLK}^{+}$cells (Jin et al., 2008; Li et al., 2015).

The relationship between expression of DLK1, PPARy and HGF needs to be studied in further, especially as marker of invasion and metastases level of HCC. HCC antimetastatic proteins, such as E-cadherin, spleen tyrosine kinase or SYK and ECM regulator metallopeptidase inhibitor 3 (TIMP3), act as anti-metastatic in HCC (Hsu and Chi 2014; Shen et al., 2012). 


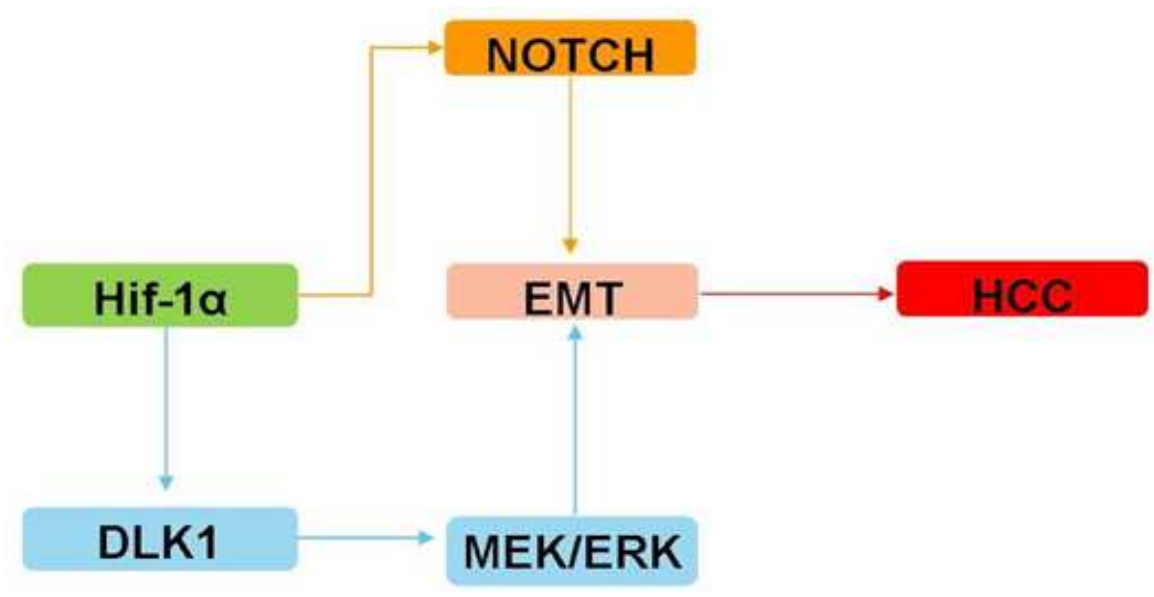

Fig. 3. Schematic diagram of DLK1 and NOTCH role in HCC oncogenesis.HIF-1 $\alpha$ and HIF-2 $\alpha$ are capable to enhance DLK1 transcription. DLK1 may increase the phosphorylation of MEK/ERK and activates MEK/ERK signaling. MEK/ERK signaling facilitates Epithelial-to-Mesenchymal Transition (EMT) process and Oct4/Nanog-regulated EMT to HCC

\section{Conclusion}

HCC and progenitor/stem cell have similar expression pattern. A protein that is found in both condition is DLK1 that can affect cell proliferation and differentiation. Some signaling pathways, such as Wnt and NOTCH pathway, are indirectly affected by DLK1; even though, the signaling pathways are not clearly understood (Fung et al., 2016). The role of DLK1 in Wnt pathway are in non-canonical or $\beta$-catenin-independent mechanism (Vilchez et al., 2016; Fung et al., 2016) and also through PPAR $\gamma$ suppression mechanism. In addition, paracrine and self-inductive effect of hepatic stellate cells were able to activate DLK1 to play a role in the process of liver development, regeneration, fibrosis and malignancy (Zhu et al., 2012; Fung et al., 2016). Further researches are expected to reveal the biological role of DLK1 in the signaling pathway. This knowledge will provide solutions for HCC therapy in the future. Another research opportunity that can be further explored is the relationship between DLK1, Wnt and PPAR $\gamma$ in oncogenesis of HCC.

\section{Acknowledgement}

Our gratitude is extended to Department of Anatomy, Faculty of Medicine, Universitas Indonesia for facilitating and supporting this study.

\section{Funding Information}

The review was funded by the grant from the Directorate of Research and Community Engangement of Universitas Indonesia, Hibah Publikasi Internasional Terindeks untuk Tugas Akhir Mahasiswa UI 2016, with the contract number of. 1916/UN2.R12/HKP.05.00/2016.

\section{Author's Contributions}

Each author has an equal contribution in the preparation, development and publication of this manuscript.

\section{Ethics}

This review does not have any ethical problems since the subject was published original articles.

\section{References}

ACS, 2016. Cancer facts and figures 2016. American Cancer Society, pp: 1-9.

Baladron, V., M.J. Ruiz-Hidalgo, M.L. Nueda, M.J. Díaz-Guerra and J.J. García-Ramírez et al., 2005. dlk acts as a negative regulator of Notch1 activation through interactions with specific EGFlike repeats. Exp. Cell Res., 303: 343-359. DOI: 10.1016/j.yexcr.2004.10.001

Begum, A., Q. Lin, C. Yu, Y. Kim and Z. Yun, 2014. Interaction of Delta-like 1 Homolog (Drosophila) with Prohibitins and Its Impact on Tumor Cell Clonogenicity. Molecular Cancer Res., 12: 155-64. DOI: 10.1158/1541-7786.MCR-13-0360

Bujak, E., D. Ritz and D. Neri, 2015. A monoclonal antibody to human DLK1 reveals differential expression in cancer and absence in healthy tissues. Antibodies, 4: 71-87. DOI: 10.3390/antib4020071

Chauhan, R. and N. Lahiri, 2016. Tissue- and serumassociated biomarkers of hepatocellular carcinoma. Biomark Cancer, 8: 37-55. DOI: 10.4137/BIC.S34413

Chen, L., D. Qanie, A. Jafari, H. Taipaleenmaki and C.H. Jensen et al., 2011. Delta-like 1/fetal antigen-1 (Dlk1/FA1) is a novel regulator of chondrogenic cell differentiation via inhibition of the Akt kinase-dependent pathway. J. Biol. Chem., 286: 32140-32149. DOI: 10.1074/jbc.M111.230110 
Coral, H., C. Wang, S. Mattu, G. Destefanis and S. Ladu et al., 2012. AKT and N-Ras co-activation in the mouse liver promotes rapid carcinogenesis via mTORC1, FOXM1/SKP2 and c-Myc pathways. Hepatology, 55: 833-45. DOI: 10.1002/hep.24736

D'Souza, B., L. Meloty-Kapella and G. Weinmaster, 2010. Canonical and non-canonical notch ligands. Current Topics Devel. Biol., 92: 73-129. DOI: 10.1016/S0070-2153(10)92003-6

Dill, M.T., L. Tornillo, T. Fritzius, L. Terracciano and D. Semela et al., 2013. Constitutive Notch2 signaling induces hepatic tumors in mice. Hepatology, 57: 1607-1619. DOI: 10.1002/hep.26165

Ding, W., H. You, H. Dang, F. LeBlanc and V. Galicia et al., 2010. Epithelial-to-mesenchymal transition of murine liver tumor cells promotes invasion. Hepatology, 52: 945-953. DOI: $10.1002 /$ hep. 23748

Falix, F.A., M.R.S. Tjon-A-Loi, I.C. Gaemers, D.C. Aronson and W.H. Lamers, 2013. DLK1 protein expression during mouse development provides new insights into its function. ISRN Dev. Biol.

DOI: $10.1155 / 2013 / 628962$

Falix, F.A., D.C. Aronson, W.H. Lamers and I.C. Gaemers, 2012. Possible roles of DLK1 in the Notch pathway during development and disease. Biochim. Biophys. Acta, 1822: 988-95. DOI: $10.1016 / \mathrm{j}$.bbadis.2012.02.003

Fung, E., H. Tsukamoto and L. Angeles, 2016. Morphogen-related therapeutic targets for liver fibrosis. Clin. Res. Hepatol Gastroenterol., 39: 1-10.

Geisler, F., F. Nagl, P.K. Mazur, M. Lee and U. ZimberStrobl et al., 2008. Liver-specific inactivation of Notch2, but not Notch1, compromises intrahepatic bile duct development in mice. Hepatology, 48: 607-616. DOI: $10.1002 /$ hep. 22381

Gordillo, M., T. Evans and V. Gouon-Evans, 2015. Orchestrating liver development. Development, 142: 2094-2108. DOI: 10.1242/dev.114215

Hansson, E.M., U. Lendahl and G. Chapman, 2004. Notch signaling in development and disease. Seminars Cancer Biol., 14: 320-328. DOI: $10.1016 /$ j.semcancer.2004.04.011

Hayashi, Y., M. Osanai and G.H. Lee, 2015. NOTCH2 signaling confers immature morphology and aggressiveness in human hepatocellular carcinoma cells. Oncol. Rep., 34: 1650-1658.

DOI: $10.3892 /$ or.2015.4171

Hsu, H.T. and C.W. Chi, 2014. Emerging role of the peroxisome proliferator-activated receptor-gamma in hepatocellular carcinoma. J. Hepatocell. Carcinoma, 1: 127-135. DOI: 10.2147/JHC.S48512

Jin, Z., R.J. Yang, B. Dong and B.C. Xing, 2008. Progenitor gene DLK1 might be an independent prognostic factor of liver cancer. Expert Opin. Biol. Ther., 8: 371-377. DOI: 10.1517/14712598.8.4.371
Jörs, S., P. Jeliazkova, M. Ringelhan, J. Thalhammer and S. Dürl et al., 2015. Lineage fate of ductular reactions in liver injury and carcinogenesis. J. Clin. Invest., 125: 2445-2457. DOI: 10.1172/JCI78585

Kim, Y., Q. Lin, D. Zelterman and Z. Yun, 2009. Hypoxia-Regulated Delta-like 1 Homologue Enhances Cancer Cell Stemness and Tumorigenicity. Cancer Res., 69: 9271-9280. DOI: 10.1158/0008-5472.CAN-09-1605

Kimura, O., Y. Kondo and T. Shimosegawa, 2012. PPAR could contribute to the pathogenesis of hepatocellular carcinoma. PPAR Res. DOI: $10.1155 / 2012 / 574180$

Kopan, R. and M.X.G. Ilagan, 2009. The canonical notch signaling pathway: Unfolding the activation mechanism. Cell, 137: 216-33. DOI: $10.1016 /$ j.cell.2009.03.045

Laborda, J., E.A. Sausville, T. Hoffman and V. Notario, 1993. dlk, a putative mammalian homeotic gene differentially expressed in small cell lung carcinoma and neuroendocrine tumor cell line. J. Biol. Chem., 268: 3817-3820. PMID: 8095043

Lee, K.H., E.Y. Choi, M.K. Kim, K.O. Kim and B.I. Jang, 2010. Inhibition of histone deacetylase activity down-regulates urokinase plasminogen activator and matrix metalloproteinase-9 expression in gastric cancer. Mol. Cell. Biochem., 343: 163-171. DOI: $10.1007 / \mathrm{s} 11010-010-0510-\mathrm{x}$

Li, H., M.L. Cui, T.Y. Chen, H.Y. Xie and Y. Cui et al., 2015. Serum DLK1 is a potential prognostic biomarker in patients with hepatocellular carcinoma. Tumor Biol., 36: 8399-8404.

Loomes, K.M., D.B. Taichman, C.L. Glover, P.T. Williams and J.E. Markowitz et al., 2002. Characterization of Notch receptor expression in the developing mammalian heart and liver. Am. J. Medical Genetics, 112: 181-189. DOI: 10.1002/ajmg.10592

Lu, J., Y. Xia, K. Chen, Y. Zheng and J. Wang et al., 2016. Oncogenic role of the notch pathway in primary liver cancer. Oncol. Lett., 12: 3-10. DOI: $10.3892 /$ ol.2016.4609

Maulik, G., A. Shrikhande, T. Kijima, P.C. Ma and P.T. Morrison et al., 2002. Role of the hepatocyte growth factor receptor, c-Met, in oncogenesis and potential for therapeutic inhibition. Cytokine Growth Factor Rev., 13: 41-59. PMID: 11750879

Mizuno, S., K. Matsumoto, M.Y. Li and T. Nakamura, 2005. HGF reduces advancing lung fibrosis in mice: A potential role for MMP-dependent myofibroblast apoptosis. FASEB J. 19: 580-582. DOI: 10.1096/fj.04-1535fje

Mohammed, F.F., C.J. Pennington, Z. Kassiri, J.S. Rubin and P.D. Soloway et al., 2005. Metalloproteinase inhibitor TIMP-1 affects hepatocyte cell cycle via HGF activation in murine liver regeneration. Hepatology, 41: 857-867. DOI: 10.1002/hep.20618 
Morell, C.M., R. Fiorotto, L. Fabris and M. Strazzabosco, 2013. Notch signalling beyond liver development: Emerging concepts in liver repair and oncogenesis. Clin. Res. Hepatol. Gastroenterol., 37: 447-454. DOI: 10.1016/j.clinre.2013.05.008

Nueda, M.L., V. Baladrón, B. Sánchez-Solana, M.Á. Ballesteros and J. Laborda, 2007. The EGF-like protein dlk1 inhibits notch signaling and potentiates adipogenesis of mesenchymal cells. J. Mol. Biol., 367: 1281-1293. DOI: 10.1016/j.jmb.2006.10.043

Ozaki, I., T. Mizuta, G. Zhao, H. Zhang and T. Yoshimura et al., 2003. Induction of multiple matrix metalloproteinase genes in human hepatocellular carcinoma by hepatocyte growth factor via a transcription factor Ets-1. Hepatol. Res., 27: 289-301. PMID: 14662117

Ross, S.E., N. Hemati, K.A. Longo, C.N. Bennett1 and P.C. Lucas et al., 2000. Inhibition of adipogenesis by Wnt signaling. Science, 289: 950-953.

DOI: $10.1126 /$ science.289.5481.950

Shen, B., E.S.H. Chu, G. Zhao, K. Man and C.W. Wu et al., 2012. PPARgamma inhibits hepatocellular carcinoma metastases in vitro and in mice. British J. Cancer, 106: 1486-1494. DOI: 10.1038/bjc.2012.130

Shen, Q., J. Fan, X.R. Yang, Y. Tan and W. Zhao et al., 2016. Serum DKK1 as a protein biomarker for the diagnosis of hepatocellular carcinoma: A large-scale, multicentre study. Lancet Oncol., 13: 817-826. DOI: $10.1016 / \mathrm{S} 1470-2045(12) 70233-4$

Shin, D., S. Pal and S. Monga, 2015. Cellular and molecular basis of liver development. Compr. Physiol., 3: 799-815. DOI: 10.1002/cphy.c120022

Sokol, R.J., 2002. Introduction to the pediatric liver research agenda: A blueprint for the future. J. Pediatric Gastroenterology Nutrition, 35: S2-S3. DOI: 10.1097/00005176-200207001-00002

Tanaka, M., M. Okabe, K. Suzuki, Y. Kamiya and Y. Tsukahara et al., 2009. Mouse hepatoblasts at distinct developmental stages are characterized by expression of EpCAM and DLK1: Drastic change of EpCAM expression during liver development. Mech. Dev., 126: 665-676. DOI: 10.1016/j.mod.2009.06.939

Tanimizu, N., M. Nishikawa, H. Saito, T. Tsujimura and A. Miyajima, 2003. Isolation of hepatoblasts based on the expression of Dlk/Pref-1. J. Cell Sci., 116: 1775-1786. DOI: $10.1242 /$ jcs.00388

Tanimizu, N. and T. Mitaka, 2014. Re-evaluation of liver stem/progenitor cells. Organogenesis, 10: 208-215. DOI: $10.4161 /$ org. 27591

Tanimizu, N., T. Tsujimura, K. Takahide, T. Kodama and K. Nakamura et al., 2004. Expression of Dlk/Pref-1 defines a subpopulation in the oval cell compartment of rat liver. Gene Expr. Patterns, 5: 209-218. DOI: 10.1016/j.modgep.2004.08.003
Teodorczyk, M. and M.H.H. Schmidt, 2014. Notching on cancer's door: Notch signaling in brain tumors. Frontiers Oncol., 4: 341-341. DOI: $10.3389 /$ fonc. 2014.00341

Vilchez, V., L. Turcios, F. Marti and R. Gedaly, 2016. Targeting Wnt/ $\beta$-catenin pathway in hepatocellular carcinoma treatment. World J. Gastroenterol., 22: 823-832. DOI: 10.3748/wjg.v22.i2.823

Wang, S.W., S.L. Pan, C.Y. Peng, D.Y. Huang and A.C. Tsai et al., 2007. CHM-1 inhibits hepatocyte growth factor-induced invasion of SK-Hep-1 human hepatocellular carcinoma cells by suppressing matrix metalloproteinase-9 expression. Cancer Lett., 257: 87-96. DOI: 10.1016/j.canlet.2007.07.002

Wang, Y. and H.S. Sul, 2006. Ectodomain shedding of preadipocyte factor 1 (Pref-1) by Tumor necrosis factor Alpha Converting Enzyme (TACE) and inhibition of adipocyte differentiation. Mol. Cell. Biol., 26: 5421-5435. DOI: 10.1128/MCB.02437-05

WHO, 2016. World Health Statistics 2016. Pp: 56-7.

Woo, H.G., E.S. Park, J.S. Lee, Y.H Lee and T. Ishikawa et al., 2009. Identification of potential driver genes in human liver carcinoma by genomewide screening. Cancer Res., 69: 4059-66. DOI: 10.1158/0008-5472.CAN-09-0164

Xu, X., R.F. Liu, X. Zhang, L.Y. Huang and F. Chen et al., 2012. DLK1 as a potential target against cancer stem/progenitor cells of hepatocellular carcinoma. Mol Cancer Therapeut., 11: 629-38. DOI: 10.1158/1535-7163.MCT-11-0531

Yin, D., D. Xie, S. Sakajiri, C.W. Miller and H. Zhu et al., 2006. DLK1: Increased expression in gliomas and associated with oncogenic activities. Oncogene, 25: 1852-1861. DOI: 10.1038/sj.onc. 1209219

Zhao, Y., R. Zhu and Y. Sun, 2016. Epithelialmesenchymal transition in liver fibrosis. Biomed. Rep., 4: 269-274. DOI: 10.3892/br.2016.578

Zhu, N.L., K. Asahina, J. Wang, A. Ueno and R. Lazaro et al., 2012. Hepatic stellate cell-derived delta-like homolog 1 (DLK1) protein in liver regeneration. J. Biol. Chem., 287: 10355-10367. DOI: $10.1074 /$ jbc.M111.312751

Zong, Y., A. Panikkar, J. Xu, A. Antoniou and P. Raynaud et al., 2009. Notch signaling controls liver development by regulating biliary differentiation. Development, 136: 1727-1739. DOI: $10.1242 /$ dev.029140

Zong, Y. and B.Z. Stanger, 2011. Molecular mechanisms of bile duct development. Int. J. Biochemistry Cell Biol., 43: 257-264. DOI: 10.1016/j.biocel.2010.06.020 